\title{
PREVENÇÃO DE AGRAVOS E PROMOÇÃO DA SAÚDE: UM ESTUDO COM TRABALHADORES PORTUÁRIOS ${ }^{1}$
}

\author{
Jorgana Fernanda de Souza Soares², Marta Regina Cezar-Vaz', Cynthia Fontella Sant'Anna
}

\begin{abstract}
${ }^{1}$ Artigo vinculado ao projeto de pesquisa intitulado - Saúde do trabalhador e conhecimento sobre situações de risco: um estudo com trabalhadores portuários avulsos do município do Rio Grande-RS e ao Programa de Pós-Graduação em Enfermagem (PPGEnf) da Universidade Federal do Rio Grande (FURG), desenvolvido no Laboratório de Estudo de Processos Socioambientais e Produção Coletiva de Saúde, 2006.

${ }^{2}$ Doutoranda em Saúde Pública pelo Programa de Pós-Graduação em Saúde Coletiva da Universidade Federal da Bahia (UFBA). Salvador, Bahia, Brasil. E-mail: jfss_rs@hotmail.com

${ }^{3}$ Doutora em Filosofia da Enfermagem. Professora Adjunto III da Escola de Enfermagem da FURG. Rio Grande, Rio Grande do Sul, Brasil. E-mail: cezarvaz@vetorial.net

${ }^{4}$ Doutoranda do Programa de Pós-Graduação em Enfermagem da FURG. Professora Assistente I da Universidade Federal do Pampa. Rio Grande, Rio Grande do Sul, Brasil. E-mail: cynthiafs_enf@yahoo.com.br
\end{abstract}

RESUMO: Estudo quantitativo, transversal e descritivo, realizado no porto do Rio Grande, Rio Grande do Sul, Brasil. Objetivou identificar o conhecimento e participação nos serviços portuários locais especializados, em amostra com 306 trabalhadores portuários avulsos. No grupo geral dos trabalhadores, a maioria identificou a existência do Programa de Prevenção de Riscos Ambientais (67,32\%), do Serviço Especializado em Segurança e Saúde no Trabalho Portuário (93,46\%) e da Comissão de Prevenção de Acidentes no Trabalho Portuário (97,38\%). Quanto à participação dos trabalhadores nos serviços citados, estratificada por categoria profissional, salienta-se, no primeiro, a colaboração, implantação, execução e respeito às orientações $(p=0,001)$; junto ao segundo e terceiro, a participação na eleição dos representantes $(\mathrm{p}=0,001)$. Evidencia-se que conhecer tais serviços e deles participar relaciona-se com a atividade desenvolvida pelos trabalhadores. As evidências apontadas contribuem para uma pactuação entre suportes educacionais e ambientais, para a gestão de riscos e a promoção da saúde dos trabalhadores portuários.

DESCRITORES: Saúde do trabalhador. Promoção da saúde. Prevenção.

\section{INJURY PREVENTION AND HEALTH PROMOTION: A STUDY AMONG DOCK WORKERS}

\begin{abstract}
The objective of this exploratory, transversal and quantitative study carried out in the port of Rio Grande, Rio Grande do Sul, Brazil is to identify the knowledge and participation of dock workers about specialized port services, involving a sample of 306 workers. In general group of dock workers, the majority identified the existence of an Environmental Risk Prevention Program (67.32\%), from the Specialized Service in Health Safety for dock workers (93.46\%) and from the dock workers Accident Prevention Commission $(97.38 \%)$. Worker participation in the services cited, stratified by professional categories, were highlighted in the first place to collaboration, implantation, execution, and respect for orientation $(\mathrm{p}=0.001)$. With respect to the second and third tasks, participation in representation election $(\mathrm{p}=0.001)$ was highlighted. This study has shown that knowing these services and participating in them is related to worker-developed activity. The evidence presented contributes to form a pact between educational and environmental supporters for dock worker risk management and health promotion
\end{abstract}

DESCRIPTORS: Occupational health. Health promotion. Prevention.

\section{PREVENCIÓN DE ENFERMEDADES Y PROMOCIÓN DE LA SALUD: UN ESTUDIO CON TRABAJADORES PORTUARIOS}

RESUMEN: Estudio cuantitativo, transversal y descriptivo, realizado en el puerto de Rio Grande, en la ciudad de Río Grande do Sul, Brasil. El objetivo fue identificar el conocimiento y participación de trabajadores al respecto de los servicios portuarios especializados, siendo utilizada una muestra con 306 individuos. En el grupo general de los trabajadores participantes del estudio, la mayoría identificó la existencia del Programa de Prevención de Riesgos Ambientales (67,32\%), del Servicio Especializado en Seguridad y Salud en el Trabajo Portuario $(93,46 \%)$ y de la Comisión de Prevención de Accidentes en el Trabajo Portuario (97,38\%). Sobre la participación de los trabajadores en los servicios citados, estratificada por categoría profesional, se destaca en relación al primero, la colaboración, implantación, ejecución y respeto a las orientaciones $(\mathrm{p}=0,001)$, y con relación al segundo y tercer servicios, la participación en la elección de los representantes ( $\mathrm{p}=0,001)$. Se evidencia que conocer estos servicios y participar se relaciona con la actividad desarrollada por los trabajadores. Tales evidencias contribuyen para un acuerdo entre el apoyo educativo y ambiental para la gestión de riesgos y la promoción de la salud de los trabajadores portuarios.

DESCRIPTORES: Salud del trabajador. Promoción de la salud. Prevención. 


\section{INTRODUÇÃO}

A Saúde do Trabalhador, enquanto incumbência da Saúde Pública brasileira, necessita adentrar em todos os ambientes de trabalho para atingir a universalidade das ações, conforme preconizam os princípios do Sistema Único de Saúde (SUS). Contudo, em grande parte das empresas, pode-se observar apenas o cumprimento das Normas Regulamentadoras (NRs) do Ministério do Trabalho e Emprego (MTE), que orientam as ações para a segurança dos trabalhadores, no aspecto de prevenção de acidentes e doenças ocupacionais. Apesar disso, considera-se que o trabalhador, quando consciente das exposições a que está submetido no ambiente de trabalho, não só atua na prevenção de doenças e acidentes, como também pode assumir a sua responsabilidade para a promoção da saúde nos espaços em que o SUS ainda não possui ações efetivas.

Claro exemplo desses espaços é o porto. O ambiente de trabalho portuário apresenta muitos fatores de risco à saúde e à vida dos trabalhadores, particularmente para os trabalhadores portuários avulsos (TPAs), que realizam o seu trabalho nos mais diversos contextos. ${ }^{1}$ Eles prestam serviços de forma eventual, sem vínculo empregatício, para várias operadoras portuárias; estas, por sua vez, solicitam a mão de obra ao Órgão Gestor de Mão de Obra $(\mathrm{OGMO})^{2}$ para o exercício das atividades de capatazia, conferência de carga, conserto de carga, estiva, trabalhadores em bloco e vigilância de embarcações.

No contexto do trabalho portuário, a NR-9 e a NR-29 são os principais instrumentos para a garantia das condições de segurança e saúde dos TPAs. A primeira se refere ao Programa de Prevenção de Riscos Ambientais (PPRA), cujo objetivo principal é a preservação da saúde e a integridade dos trabalhadores; para alcançá-lo, necessita da participação dos TPAs para a implantação e a execução; o respeito às orientações recebidas nos treinamentos oferecidos dentro do Programa e a informação imediata aos responsáveis dos incidentes que possam representar risco à saúde dos trabalhadores. ${ }^{3}$ Já a NR-29 regulamenta as ações necessárias para a preservação da segurança e da saúde dos trabalhadores portuários, instituindo o Serviço Especializado em Segurança e Saúde no Trabalho Portuário (SESSTP) e a Comissão de Prevenção de Acidentes no Trabalho Portuário (CPATP). O SESSTP possui como principais atribuições promover e proteger a saúde do trabalhador no ambiente portuário. ${ }^{4}$ A CPATP objetiva observar e relatar condições de risco nos ambientes de trabalho e solicitar medidas de diminuição/ eliminação dos fatores de risco existentes; discutir os acidentes ocorridos, enviando ao SESSTP, OGMO ou aos empregadores o resultado da discussão; solicitar medidas de prevenção de acidentes semelhantes aos já ocorridos e orientar os trabalhadores quanto à prevenção de acidentes ${ }^{4}$ com a participação dos mesmos. De acordo com a NR-29, eles participam das ações desenvolvidas pelo SESSTP e CPATP, através da eleição dos seus representantes na CPATP, indicando a ambos as situações de risco, sugerindo melhorias nas condições de trabalho, cumprindo as recomendações para a prevenção de acidentes e comparecendo às reuniões da CPATP, sempre que convocado. ${ }^{4}$

Partindo desta breve exposição, é possível visualizar o caráter majoritariamente preventivo do PPRA, SESSTP e CPATP; porém, a prevenção aqui referida é capaz de subsidiar a promoção da saúde dos TPAs, por se entender que a mesma pode ser definida como uma pactuação entre os suportes educacionais e ambientais, visando atingir ações e condições de vida que direcionem à saúde. ${ }^{5-6}$ Sendo assim, o conhecimento de tais instâncias por parte do trabalhador remete ao foco pretendido, que identifica a relação entre o conhecer e o atuar no ambiente de trabalho, com vistas a promover condições saudáveis. Visando à promoção da saúde por meio das ações de prevenção de doenças, a enfermagem possui um papel fundamental, por constituir as equipes multiprofissionais do SESSTP. No âmbito da saúde do trabalhador, uma das possibilidades de aplicação do conhecimento clínico das enfermeiras, assume-se que a prevenção de doenças com o intuito de adotar comportamentos mais saudáveis tem o seu limite nos aspectos culturais dos indivíduos focados nas ações de saúde. ${ }^{7}$ Nesse escopo, o conhecimento dos trabalhadores sobre as possibilidades de prevenção de doenças e de promoção da saúde por meio de mecanismos organizacionais instituídos, pode se transformar em instrumentos de trabalho para a enfermagem.

A relevância da temática do presente estudo reside na pouca exploração do ambiente portuário como objeto de pesquisas, assim como na necessidade de se conhecer o que é realizado a fim de que a Saúde do Trabalhador no SUS possa reconhecer o cenário como sendo de sua responsabilidade e ainda na particularidade da possibilidade da enfermagem atuar no setor em questão. O trabalho ora apresentado objetivou identificar o conhecimento dos TPAs acerca da existência e das 
atribuições do PPRA, do SESSTP e da CPATP, bem como suas formas de participação nessas ações por atividade profissional.

\section{MÉTODO}

Trata-se de uma pesquisa quantitativa, de corte transversal, descritivo, realizada no porto marítimo do Rio Grande, Rio Grande do Sul, Brasil, no primeiro semestre de 2006.

No porto do Rio Grande, conforme os dados obtidos junto ao OGMO-Rio Grande (OGMO-RG), a população efetiva de trabalhadores portuários avulsos, na época temporal da pesquisa, era de 758 trabalhadores.

Foi realizado um estudo com trinta TPAs, com o propósito de realizar um diagnóstico situacional sobre o seu conhecimento dos riscos presentes no ambiente de trabalho, devido à indisponibilidade de dados acerca do objeto do estudo. Os participantes foram selecionados conforme a sua disposição para responder à duas perguntas abertas sobre a existência de riscos no ambiente de trabalho e quais eram estes.

Após este estudo, a amostra foi calculada utilizando-se o nível de confiança de 95\%, com a prevalência de conhecimento dos riscos à saúde estimada a partir do diagnóstico situacional em $93 \%$, com precisão de $+-3 \%$. Assim, obteve-se o $\mathrm{n}=278$ e, considerando $10 \%$ de perdas, a amostra calculada foi de 306 TPAs. Os trabalhadores foram subdivididos em subamostras de acordo com a proporção de TPAs em cada categoria profissional. Participaram do estudo 138 (45,25\%) trabalhadores da capatazia; 18 (5,8\%) conferentes de carga; quatro $(1,19 \%)$ consertadores de carga; 132 (43,19\%) estivadores; três $(1,06 \%)$ trabalhadores em bloco e onze (3,56\%) vigilantes de embarcações. A amostra da pesquisa é do tipo não-aleatória, sendo que participaram do estudo os trabalhadores portuários avulsos que haviam se apresentado para concorrer a uma oportunidade de trabalho no turno.

A entrevista estruturada utilizada para a coleta dos dados foi construída a partir dos resultados obtidos no diagnóstico situacional, complementada pela revisão de literatura e observações espontâneas dos pesquisadores no porto. Foi realizado o estudo piloto para testar a entrevista.

O questionário para a coleta de dados é misto, constituído por variáveis qualitativas nominais e por quantitativas discretas. A coleta dos dados foi realizada no primeiro e segundo semestre de
2006, nas proximidades dos locais onde ocorre a escalação dos trabalhadores para o turno de trabalho, através da aplicação da entrevista estruturada. Realizou-se o teste a de Crombach para medir a fidedignidade do instrumento e o resultado obtido foi de 0,73 e 0,92, respectivamente. Os instrumentos foram revisados para averiguar e corrigir possíveis erros de codificação.

As variáveis selecionadas para o presente trabalho foram as sóciodemográficas: sexo, idade, tempo de atuação no setor, grau de instrução, estado civil, cor e rendimento financeiro - e a variável principal foi o conhecimento dos TPAs da existência e das funções do PPRA, SESSTP e CPATP e as suas formas de participação nas referidas ações: realizar cursos e informar aos responsáveis os riscos e os acidentes.

Os dados foram digitados duplamente no programa EPINFO 6.04d (Centers for Disease Control and Prevention, Atlanta, Estados Unidos), para a limpeza do banco e, posteriormente, transportados para outro software estatístico, onde se realizou análise descritiva das variáveis qualitativas nominais e análise de variância amostral seguida do teste Post hoc do Least Square Diference (LSD) de Fisher ou Teste $T$ de Student para as variáveis quantitativas discretas, com o nível de significância $a=0,05$. Foram imputados os consertadores de carga e os trabalhadores em bloco, da análise de variância estratificada, para evitar o erro na estimativa das associações estatísticas, devido ao pequeno número de trabalhadores entrevistados nas referidas atividades.

Em relação aos aspectos éticos da pesquisa, respeitando a resolução do CNS 196/ $96,{ }^{8}$ foi solicitado e recebido o consentimento do OGMO-RG para a realização do estudo junto aos TPAs. O trabalho foi aprovado pelo Comitê de Ética em Pesquisa na Área da Saúde da Universidade Federal do Rio Grande (Processo no 23116.6779/2005-98) e foi utilizado o consentimento livre e esclarecido do participante, obtido previamente à entrevista, assinado em duas vias, permanecendo uma com a entrevistadora, para arquivamento junto ao processo da pesquisa, e a outra, com o participante.

\section{RESULTADOS}

Todos os TPAs eram do sexo masculino, $32,03 \%$ tinham entre 49 e 58 anos de idade e $39,22 \%$ trabalhavam no porto há um período que varia entre 20 e 34 anos. Dos 294 (96,08\%) respondentes da questão referente aos rendimentos financei- 
ros, 40,20\% receberam de dois a quatro salários-mínimos (Tabela 1). Ainda na tabela 1, pode-se observar que $35,95 \%$ dos trabalhadores tinham o Ensino Fundamental incompleto, 57,84\% eram casados ou viviam em união consensual e $57,84 \%$ se autoavaliaram como brancos.

Tabela 1 - Características sócio-demográficas dos trabalhadores portuários avulsos (n=306). Rio Grande-RS, Brasil, 2006

\begin{tabular}{|c|c|c|}
\hline Variável & $\begin{array}{c}\text { Número de } \\
\text { trabalhadores }\end{array}$ & $\begin{array}{c}\text { Proporção de } \\
\text { trabalhadores (\%) }\end{array}$ \\
\hline \multicolumn{3}{|l|}{ Idade (ano) } \\
\hline $19-28$ & 35 & 11,44 \\
\hline $29-38$ & 77 & 25,16 \\
\hline $39-48$ & 85 & 27,78 \\
\hline $49-58$ & 98 & 32,03 \\
\hline $59-68$ & 11 & 3,59 \\
\hline \multicolumn{3}{|l|}{ Tempo de atuação (ano) } \\
\hline$<$ de 02 & 07 & 2,29 \\
\hline $02-04$ & 52 & 16,99 \\
\hline $05-09$ & 12 & 3,92 \\
\hline $10-19$ & 95 & 31,05 \\
\hline $20-34$ & 120 & 39,22 \\
\hline$>35$ anos & 20 & 6,54 \\
\hline \multicolumn{3}{|l|}{ Rendimento financeiro (salário mínimo)* } \\
\hline Menos de 2 & 15 & 4,90 \\
\hline $2-4$ & 123 & 40,20 \\
\hline $5-9$ & 78 & 25,49 \\
\hline$>10$ & 78 & 25,49 \\
\hline Ignorado & 12 & 3,92 \\
\hline \multicolumn{3}{|l|}{ Grau de instrução } \\
\hline Analfabeto & 02 & 0,65 \\
\hline Ensino fundamental incompleto & 110 & 35,95 \\
\hline Ensino fundamental completo & 55 & 17,98 \\
\hline Ensino médio incompleto & 34 & 11,11 \\
\hline Ensino médio completo & 88 & 28,76 \\
\hline Ensino superior incompleto, completo, pós-graduação & 17 & 5,56 \\
\hline \multicolumn{3}{|l|}{ Estado civil } \\
\hline Solteiro & 87 & 28,43 \\
\hline Casado/união consensual & 174 & 56,87 \\
\hline Separado/desquitado/divorciado & 40 & 13,07 \\
\hline Viúvo & 05 & 1,63 \\
\hline \multicolumn{3}{|l|}{ Cor da pele } \\
\hline Branco & 177 & 57,84 \\
\hline Pardo & 57 & 18,63 \\
\hline Preto & 50 & 16,34 \\
\hline Indígena & 21 & 6,86 \\
\hline Amarelo & 01 & 0,33 \\
\hline
\end{tabular}

Do total de entrevistados, $67,32 \%$ reconheceu a existência do PPRA, 93,46\% do SESSTP e 97,38\% da CPATP. Na tabela 2, observa-se a média o desvio-padrão e a análise de variância das variáveis referentes ao conhecimento dos TPAs sobre a finalidade do PPRA, SESSTP e CPATP e as suas formas de participação nessas ações. Para o conhecimento dos trabalhadores acerca de suas atribuições no PPRA, a média atribuída ao item informar aos responsáveis os incidentes que possam representar riscos à saúde dos trabalhadores $(8,54 \pm 2,75)$ foi maior do que a dos itens 
respeitar as orientações recebidas nos treinamentos oferecidos dentro do Programa $(7,97 \pm 3,11)$ e colaborar na implantação e execução do Programa $(7,11 \pm 3,30)$, estatisticamente significante $(p=0,00)$. Observa-se ainda que nas atribuições do SESSTP, o quesito promover a saúde do trabalhador no ambiente de trabalho $(7,76 \pm 2,69)$ não foi diferen- temente avaliado $(p=0,50)$ com relação a proteger a saúde do trabalhador no ambiente de trabalho $(7,84 \pm 2,76)$. Ainda na tabela 2 , visualiza-se que a principal finalidade da CPATP, para os TPAs, é a de orientá-los em relação à prevenção de acidentes $(8,10 \pm 2,54)$, com diferença estatisticamente significante $(p=0,00)$ das demais atribuições.

Tabela 2 - Conhecimento dos trabalhadores portuários avulsos sobre a finalidade dos serviços especializados e as suas formas de participação junto a essas ações (n=306). Rio Grande-RS, Brasil, 2006

\begin{tabular}{|c|c|c|}
\hline Variável & 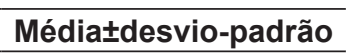 & $\mathbf{p}^{*}$ \\
\hline Forma de participação do TPA no PPRA & & 0,001 \\
\hline Colaborar na implantação e na execução do programa & $7,11 \pm 3,30$ & C \\
\hline Respeitar as orientações recebidas dentro do programa & $7,97 \pm 3,11$ & B \\
\hline $\begin{array}{l}\text { Informar aos responsáveis as situações que possam representar risco à saúde dos } \\
\text { trabalhadores }\end{array}$ & $8,54 \pm 2,75$ & A \\
\hline Finalidade da SESSTP & & 0,50 \\
\hline Promover a saúde do trabalhador no trabalho ${ }^{\dagger}$ & $7,76 \pm 2,69$ & --- \\
\hline Proteger a saúde do trabalhador no trabalho ${ }^{\dagger}$ & $7,84 \pm 2,76$ & -- \\
\hline Finalidade da CPATP & & 0,001 \\
\hline Observar e informar condições de risco no trabalho & $7,62 \pm 2,92$ & B \\
\hline Solicitar medidas de diminuição/eliminação dos riscos existentes ${ }^{\dagger}$ & $7,66 \pm 2,76$ & B \\
\hline $\begin{array}{l}\text { Discutir os acidentes ocorridos, encaminhando ao SESSTP, ao OGMO ou aos } \\
\text { empregadores o resultado da discussão }{ }^{\dagger}\end{array}$ & $7,07 \pm 3,20$ & C \\
\hline Solicitar medidas preventivas para os acidentes semelhantes aos já ocorridos ${ }^{\dagger}$ & $7,54 \pm 2,88$ & B \\
\hline Orientar os trabalhadores em relação à prevenção de acidentes ${ }^{\dagger}$ & $8,10 \pm 2,54$ & A \\
\hline Forma de participação do TPA na CPATP e SESSTP & & 0,001 \\
\hline Eleger seus representantes (CPATP) & $8,35 \pm 2,64$ & $\mathrm{~B}$ \\
\hline Indicar a elas situações de risco, sugerindo melhorias nas condições de trabalho & $8,08 \pm 2,93$ & B \\
\hline Cumprir as recomendações para a prevenção de acidentes por elas fornecidas & $8,82 \pm 2,11$ & A \\
\hline Comparecer às reuniões da CPATP, sempre que convocado & $6,50 \pm 4,01$ & C \\
\hline
\end{tabular}

${ }^{*}$ Letras diferentes representam médias estatisticamente diferentes $(\mathrm{p}<0,05) ; \dagger \mathrm{n}=305$ respondentes.

Na tabela 3, observa-se a médiat desvio-padrão e a probabilidade do teste LSD de Fisher das variáveis quantitativas discretas, referentes ao conhecimento do TPA sobre as atribuições do PPRA, SESSTP e CPATP e a sua forma de participação junto a tais ações, estratificadas por atividade. A função do TPA de colaborar na implantação e na execução do PPRA foi bastante diferente $(p=0,001)$ entre as atividades profissionais. Recebeu nota média significativamente superior dos trabalhadores em capatazia $(7,49 \pm 3,12)$ e estiva $(7,14 \pm 3,25)$ se comparada com a dos conferentes de carga $(4,33 \pm 3,87)$. A função respeitar as orientações recebidas dentro do Programa também foi avaliada de maneira estatisticamente diferente $(p=0,001)$ pelas atividades dos TPAs. Recebeu maior nota média da estiva $(8,26 \pm 2,85)$ e dos trabalhadores em capatazia $(8,07 \pm 2,99)$, na comparação com os conferentes de carga $(5,28 \pm 4,34)$. A nota média atribuída a informar aos responsáveis os incidentes que possam representar riscos à saúde dos trabalhadores foi avaliada de maneira também distinta $(p=0,001)$ entre as atividades profissionais. As notas médias atribuídas pelos trabalhadores em estiva $(8,76 \pm 2,51)$ e capatazia $(8,74 \pm 2,44)$ foram maiores do que as apontadas pelos conferentes de carga $(6,06 \pm 4,33)$.

A finalidade do SESSTP promover a saúde do trabalhador no ambiente de trabalho foi avaliada de maneira significativamente diferente $(p=0,001)$ entre as atividades dos TPAs. A nota média atribuída pelos trabalhadores em capatazia $(8,03 \pm 2,31)$ foi significativamente superior à dada pelos vigilantes de embarcações $(6,18 \pm 2,86)$ e conferentes de carga $(5,71 \pm 3,82)$. A outra atribuição do SESSTP, referente à proteção da saúde do trabalhador no ambiente de trabalho, também foi avaliada de maneira significativamente diferente $(p=0,001)$ entre as atividades profissionais. Os trabalhadores em capatazia $(8,15 \pm 2,40)$ e estiva $(7,79 \pm 2,74)$ atribuíram 
maior nota média do que os conferentes de carga $(6,12 \pm 3,64)$ e vigilantes de embarcações $(5,09 \pm 3,21)$.

A atribuição orientar os trabalhadores em relação à prevenção de acidentes da CPATP foi avaliada de maneira significativamente diferente $(p=0,02)$ entre as atividades. Recebeu nota média significativamente superior dos trabalhadores em capatazia $(8,22 \pm 2,34)$ e estiva $(8,21 \pm 2,58)$ do que a atribuída pelos conferentes de carga $(6,94 \pm 3,59)$ e vigilantes de embarcações $(6,27 \pm 2,15)$.

Tabela 3 - Conhecimento do trabalhador portuário avulso sobre as atribuições dos serviços especializados e a sua forma de participação junto a essas ações, estratificado por atividade $(n=299)$. Rio Grande-RS, Brasil, 2006

\begin{tabular}{|c|c|c|c|c|c|}
\hline \multirow[b]{2}{*}{ Variável } & \multicolumn{4}{|c|}{ Atividade* } & \multirow[b]{2}{*}{ p } \\
\hline & Capatazia & Estiva & $\begin{array}{l}\text { Conferência } \\
\text { de carga }\end{array}$ & $\begin{array}{l}\text { Vigilância de } \\
\text { embarcação }\end{array}$ & \\
\hline \multicolumn{6}{|l|}{ Forma de participação do TPA no PPRA } \\
\hline $\begin{array}{l}\text { Colaborar na implantação e na execução do } \\
\text { programa }\end{array}$ & $7,49 \pm 3,12^{\mathrm{a}}$ & $7,14 \pm 3,25^{a}$ & $4,33 \pm 3,87^{b}$ & $5,91 \pm 2,81^{\text {ab }}$ & 0,001 \\
\hline $\begin{array}{l}\text { Respeitar as orientações recebidas nos treina- } \\
\text { mentos recebidos dentro do programa }\end{array}$ & $8,07 \pm 2,99^{a}$ & $8,26 \pm 2,85^{a}$ & $5,28 \pm 4,34^{\mathrm{b}}$ & $7,55 \pm 3,45^{\mathrm{ab}}$ & 0,001 \\
\hline $\begin{array}{l}\text { Informar aos responsáveis os incidentes que pos- } \\
\text { sam representar riscos à saúde dos trabalhadores }\end{array}$ & $8,74 \pm 2,44^{a}$ & $8,76 \pm 2,51^{\mathrm{a}}$ & $6,06 \pm 4,33^{b}$ & $7,73 \pm 3,77^{\mathrm{ab}}$ & 0,0001 \\
\hline \multicolumn{6}{|l|}{ Finalidade da SESSTP } \\
\hline $\begin{array}{l}\text { Promover a saúde do trabalhador no ambiente de } \\
\text { trabalho }{ }^{+}\end{array}$ & $8,03 \pm 2,31^{a}$ & $7,79 \pm 2,78^{\mathrm{ab}}$ & $5,71 \pm 3,82^{c}$ & $6,18 \pm 2,86^{\text {bc }}$ & 0,001 \\
\hline $\begin{array}{l}\text { Proteger a saúde do trabalhador no ambiente de } \\
\text { trabalho }^{\dagger}\end{array}$ & $8,15 \pm 2,40^{a}$ & $7,95 \pm 2,74^{a}$ & $6,12 \pm 3,64^{b}$ & $5,09 \pm 3,21^{b}$ & 0,0001 \\
\hline \multicolumn{6}{|l|}{ Finalidade da CPATP } \\
\hline $\begin{array}{l}\text { Observar e informar condições de risco no ambi- } \\
\text { ente de trabalho }\end{array}$ & $7,63 \pm 2,82$ & $7,80 \pm 2,88$ & $6,78 \pm 3,57$ & $5,64 \pm 3,44$ & 0,72 \\
\hline $\begin{array}{l}\text { Solicitar medidas de diminuição/eliminação dos } \\
\text { riscos existentes }{ }^{\ddagger}\end{array}$ & $7,62 \pm 2,75$ & $7,76 \pm 2,72$ & $7,17 \pm 3,57$ & $6,91 \pm 2,66$ & 0,67 \\
\hline $\begin{array}{l}\text { Discutir os acidentes ocorridos enviando ao SES- } \\
\text { STP, ao OGMO ou aos empregadores o resultado } \\
\text { da discussão }{ }^{\ddagger}\end{array}$ & $7,09 \pm 3,25$ & $7,12 \pm 3,16$ & $6,11 \pm 3,68$ & $6,45 \pm 2,98$ & 0,58 \\
\hline $\begin{array}{l}\text { Solicitar medidas preventivas para acidentes } \\
\text { semelhantes aos já ocorridos }{ }^{\ddagger}\end{array}$ & $7,69 \pm 2,62$ & $7,50 \pm 3,02$ & $7,00 \pm 3,66$ & $6,18 \pm 3,46$ & 0,34 \\
\hline $\begin{array}{l}\text { Orientar os trabalhadores a respeito da prevenção } \\
\text { de acidentes }{ }^{\ddagger}\end{array}$ & $8,22 \pm 2,34^{a}$ & $8,21 \pm 2,58^{\mathrm{a}}$ & $6,94 \pm 3,59^{b}$ & $6,27 \pm 2,15^{b}$ & 0,02 \\
\hline \multicolumn{6}{|l|}{$\begin{array}{l}\text { Forma de participação do TPA na CPATP e } \\
\text { SESSTP }\end{array}$} \\
\hline Eleger seus representantes na CPATP & $8,54 \pm 2,24^{a}$ & $8,56 \pm 2,52^{\mathrm{a}}$ & $5,00 \pm 3,99^{b}$ & $8,09 \pm 3,24^{a}$ & 0,0001 \\
\hline $\begin{array}{l}\text { Indicar a SESSTP e CPATP situações de riscos e } \\
\text { sugerir melhorias nas condições de trabalho }\end{array}$ & $8,22 \pm 2,58^{a}$ & $8,23 \pm 3,01^{\mathrm{a}}$ & $5,78 \pm 4,01^{b}$ & $7,82 \pm 3,43^{\mathrm{ab}}$ & 0,01 \\
\hline $\begin{array}{l}\text { Cumprir as orientações recebidas nos treinamen- } \\
\text { tos oferecidos pela CPATP e SESSTP }\end{array}$ & $9,09 \pm 1,68^{a}$ & $8,88 \pm 2,05^{a}$ & $6,67 \pm 3,68^{b}$ & $8,00 \pm 2,90^{\mathrm{ab}}$ & 0,001 \\
\hline $\begin{array}{l}\text { Comparecer às reuniões da CPATP, sempre que } \\
\text { convocado }\end{array}$ & $7,01 \pm 3,77$ & $6,12 \pm 4,16$ & $4,83 \pm 4,13$ & $6,27 \pm 4,45$ & 0,09 \\
\hline
\end{tabular}

\footnotetext{
* Os valores representam a média \pm desvio-padrão estratificados por atividade. Letras diferentes representam médias estatisticamente diferentes $(\mathrm{p}<0,05) ; \dagger \mathrm{n}$ conferência de carga $=17 ; \ddagger \mathrm{n}$ estiva $=131$.
}

Os TPAs reconheceram como sua principal atribuição junto à CPATP e ao SESSTP a participação na eleição dos seus representantes na CPATP, que foi avaliada de maneira significativamente diferente $(p=0,001)$ entre as categorias de TPAs. Os estivadores $(8,56 \pm 2,52)$, trabalhadores em capatazia $(8,54 \pm 2,24)$ e vigilantes de embarcações $(8,09 \pm 3,24)$ atribuíram nota média significativamente maior às dadas pelos conferentes de carga $(5,00 \pm 3,99)$. A função indicar à CPATP e ao SESSTP situações de risco e sugerir melhorias nas condições de trabalho também foi avaliada significati- 
vamente diferente $(p=0,01)$ entre as atividades. Recebeu nota média significativamente diferente dos estivadores $(8,23 \pm 3,01)$ e trabalhadores em capatazia $(8,22 \pm 2,58)$, se comparada à dos conferentes de carga $(5,78 \pm 4,01)$. A atribuição cumprir as recomendações para a prevenção de acidentes nos treinamentos fornecidos pela CPATP e SESS$\mathrm{TP}$, também foi avaliada de maneira diferente $(p=0,001)$ entre as atividades de TPAs. Recebeu nota média superior dos trabalhadores em capatazia $(9,09 \pm 1,68)$ e estiva $(8,88 \pm 2,05)$, em relação aos conferentes de carga $(6,67 \pm 3,68)$.

\section{DISCUSSÃO}

O PPRA foi concluído e implantado em maio de $1999 .{ }^{9}$ O programa visa preservar a saúde e a integridade dos trabalhadores, através da antecipação, reconhecimento, avaliação e controle dos riscos ambientais existentes ou que possam vir a existir no contexto de trabalho, considerando a proteção do meio ambiente e dos recursos naturais. ${ }^{5}$ Aqui, pode-se observar o caráter preventivo das ações desenvolvidas no porto, segundo o qual a exposição é considerada como um elemento externo, previsível e dependente das atitudes do trabalhador. Mas tal prevenção pode viabilizar a promoção da saúde do trabalhador, a partir do momento em que contribui para a construção do saber do TPA acerca dos riscos à sua saúde e à do ambiente, podendo torná-lo atuante para sua autopreservação e também para a preservação do ecossistema circunvizinho.

Nesse cenário, onde a prevenção dos agravos ocupacionais pode viabilizar a promoção da saúde através da participação dos trabalhadores, existe o SESSTP, o qual foi implantado em dezembro de $1997^{9}$ e, conforme é possível constatar, atualmente é reconhecido pelos TPAs. A promoção e a proteção da saúde, em consonância com as notas médias atribuídas pelo grupo geral de trabalhadores, são funções igualmente importantes do SESSTP. Os profissionais do Serviço devem realizar identificação prévia da situação de segurança a bordo das embarcações e dos equipamentos e acessórios que serão utilizados na operação portuária, visando à prevenção de doenças e de acidentes de trabalho. ${ }^{4}$ Pode viabilizar a promoção da saúde dos trabalhadores a partir das informações prestadas sobre a infraestrutura da embarcação, do cais e as medidas necessárias para o autocuidado. Salienta-se que, em consonância com a NR 29, é possível afirmar que o SESSTP atua principalmente na prevenção de doenças e se detém a antecipar ações com o intuito de controlar agravos específicos; é estruturado para normatizar o que é ou não saudável. Além do que, o reconhecimento da existência da CPATP, eleita e empossada em 1998, ${ }^{9}$ auxilia os trabalhadores a interagirem com os órgãos empresariais, para que sejam efetivadas ações de prevenção que viabilizem a promoção da saúde.

O reconhecimento do TPA, de sua função junto ao PPRA ser a de informar aos responsáveis os incidentes que possam representar riscos à saúde dos trabalhadores talvez esteja atrelado, segundo julgamento próprio, a situações que possam representar fatores de risco imediatos à segurança e à saúde dos trabalhadores. Assim, pode-se afirmar que, no conhecimento dos trabalhadores acerca de suas funções dentro do PPRA, evidencia-se o que é mais importante em curto prazo, no que se refere a sua saúde ou vida; procura sanar tal necessidade por meio da comunicação aos responsáveis acerca das exposições iminentes. Essa atitude pode levá-los à coparticipação social, na direção de viabilizar as mudanças necessárias para tornar o ambiente portuário menos insalubre e perigoso.

A CPATP, de acordo com a nota média atribuída pelo grupo geral, possui como função mais relevante orientar os trabalhadores em relação à prevenção de acidentes. Talvez isso possa estar vinculado ao que é realizado com os trabalhadores, porque as demais funções que, a saber, são: observação e relato das condições de risco no contexto de trabalho, apontando medidas de redução; eliminação ou neutralização dos riscos existentes; discussão dos acidentes ocorridos, cujo resultado é enviado ao SESSTP, ao OGMO ou aos empregadores; solicitação de medidas de prevenção a acidentes semelhantes aos já ocorridos ${ }^{4}$ provavelmente sejam realizadas internamente com os integrantes da Comissão e o OGMO, não chegando ao conhecimento dos outros trabalhadores.

O SESSTP, conforme já mencionado, visa à prevenção de doenças e acidentes no ambiente portuário. ${ }^{4}$ A partir das notas médias atribuídas pelos trabalhadores, a finalidade em pauta foi mais reconhecida pelos trabalhadores em capatazia e estiva do que pelos conferentes de carga e vigilantes de embarcações. Talvez o resultado obtido possa demonstrar um maior conhecimento, por parte das duas primeiras categorias, viabilizando a esses numerosos grupos uma maior interação com os trabalhadores do SESSTP, visando a melhores condições de segurança nas operações, seja no cais ou a bordo das embarcações. 
A colaboração para implantar e executar o PPRA pode representar uma maneira de os trabalhadores se instrumentalizarem, mesclando os conhecimentos construídos no fazer cotidiano com os científicos, possibilitando a organização de novos saberes e, consequentemente, o reconhecimento por parte do trabalhador acerca de si próprio, na condição de sujeito ativo na transformação de situações de risco à saúde no contexto portuário, criando, assim, um ambiente saudável para os TPAs e a população circunvizinha. Pode-se afirmar que tal possibilidade se apresenta mais aos trabalhadores em capatazia e estiva, provavelmente por sua maior interação com a equipe do SESSTP.

A observância das informações e do conhecimento produzido através das orientações recebidas dentro do PPRA pode representar, no ambiente portuário, uma das maneiras possíveis de preservação do ser humano, não somente do trabalhador, mas de todos os envolvidos direta ou indiretamente com as águas que banham o porto e também uma forma responsável de preservar a vida no ecossistema que o cerca e no qual se inclui. As manifestações recolhidas podem se apresentar mais próximas dos estivadores e dos trabalhadores em capatazia, se comparadas aos conferentes de carga, porque os últimos trabalhadores, devido às próprias características da sua função nas operações representam o "patrão-simbólico", responsáveis diretos pela produção, o que talvez possa conduzi-los a um isolamento e à não participação direta nas trocas produzidas nas relações intersubjetivas, as quais, por sua vez, colaboram na construção dos saberes no cotidiano do trabalho portuário. Assim, é oportuno considerar que os conferentes de carga possuem uma identidade diferente dos demais trabalhadores, porque "a identidade se reconhece e reproduz como idêntico, mas sempre em contraste com 'o outro', num processo de ressignificação e reconstituição de sua singularidade e sua especificidade" ${ }^{10: 278}$

O fato de a finalidade do SESSTP promover a saúde do trabalhador no ambiente de trabalho ter sido melhor avaliada pelos trabalhadores em capatazia pode representar que a intervenção cotidiana dos profissionais do SESSTP, possivelmente, viabilize a promoção da saúde dos TPAs, através da interação entre aqueles e os trabalhadores avulsos, considerando os saberes tanto dos profissionais formados em segurança quanto dos TPAs, ou seja, sem desconsiderar o conhecimento construído nas relações produtivas. Isso por se entender que existe um saber peculiar ao trabalhador e ao seu grupo, assumindo-se que, nas interações sociais expressas pela produção da existência do trabalhador de maneira individual ou coletiva, ele produz conhecimento, detém um saber e tem uma determinada consciência da realidade. ${ }^{11}$

A atribuição do TPA junto ao SESSTP e à CPATP ser a de informar aos responsáveis os incidentes que possam representar riscos à saúde dos trabalhadores evidencia a importância do saber do TPA a respeito dos fatores de risco aos quais está exposto no ambiente de trabalho e dos responsáveis pela segurança e saúde, já que "saber é conhecer, ser capaz de distinguir". ${ }^{12: 125}$ O conhecimento, independentemente de ter sido construído pelo trabalhador em ambientes de educação formal, como as escolas, ou em ambientes de educação não formal, como o próprio contexto de trabalho portuário ou nos cursos oferecidos sobre as exposições ocupacionais, é de extrema importância para a prevenção dos agravos ocupacionais e para a promoção da saúde. Sem o conhecimento, o trabalhador é incapaz de identificar quais são as situações em que a insalubridade (fator de risco à saúde) se transforma em periculosidade (fator de risco à vida) e informar aos responsáveis pela operação portuária sobre tais eventos, com o objetivo de diminuir/neutralizar a exposição, prevenindo acidentes e possibilitando o impulso à transformação social. A partir do exposto e expresso pelas notas médias atribuídas pelos TPAs, pode-se afirmar que os trabalhadores em estiva e capatazia possuem um conhecimento mais elaborado acerca da função em estudo do que os conferentes de carga, o que pode representar um maior comprometimento dos estivadores e trabalhadores em capatazia com a sua própria saúde e com a de seus colegas. Em relação ao conhecimento do TPA acerca das suas funções relativas à CPATP e ao SESSTP, no grupo geral de trabalhadores, o quesito cumprir as recomendações para a prevenção de acidentes recebeu nota média significativamente superior aos demais itens. Possivelmente isso seja resultante do trabalho constante dos setores de segurança e de saúde do trabalho portuário, das pessoas que estão comprometidas com o SESSTP, a CPATP e com os TPAs diariamente. Além disso, o fato de a maior nota média ser a da variável focada permite afirmar o reconhecimento, por parte dos trabalhadores, daquilo que se apresenta em seu cotidiano, o que pode fazer com que eles não se vejam como corresponsáveis em relação à sua saúde e segurança.

A função dos TPAs eleger os seus representantes na CPATP foi avaliada significativamente diferente entre as categorias de TPAs. A partir daí, 
é possível entender que os estivadores, os trabalhadores em capatazia e os vigilantes de embarcações visualizam-se como mais responsáveis pela participação das eleições da CPATP, demonstrando um maior exercício da cidadania no ambiente de trabalho, quando comparados aos conferentes de carga, talvez porque os últimos não possuam representantes dentro da CPATP. Salienta-se que, na América Latina, a cidadania está relacionada à reprodução da força de trabalho; assim, "neste sentido, poder-se-ia dizer que o grau de cidadania de uma população varia de acordo com sua capacidade de garantir maior ou menor qualidade na reprodução da sua força de trabalho". ${ }^{13: 32}$

O fato de que indicar à CPATP e ao SESSTP situações de risco e sugerir melhorias nas condições de trabalho ter sido avaliado de maneira diferente entre as atividades talvez evidencie que os trabalhadores em estiva e capatazia percebam-se mais aderidos ao trabalho enquanto coletivo, devido às próprias características das suas equipes nas operações portuárias, provavelmente por serem mais numerosos nas referidas operações que os conferentes de carga.

Enfatiza-se que as funções do TPA em relação à CPATP e ao SESSTP, que compreendem eleger os seus representantes na CPATP e comunicar as situações de risco e sugerir melhorias nas condições de trabalho, podem representar ações de promoção da cidadania no ambiente portuário, consequentemente, propiciando que as ações de prevenção dos agravos à saúde do trabalhador avancem para a promoção da saúde.

O quesito cumprir as recomendações para a prevenção de acidentes fornecidas pela CPATP e SESSTP também foi avaliado de maneira diferente entre as categorias de TPAs. Os trabalhadores em capatazia e estiva, conforme o referido anteriormente, estão em maior número nas operações portuárias, favorecendo as trocas diárias com os trabalhadores doSESSTP e com os representantes da CPATP, o que pode contribuir para a construção do conhecimento acerca da necessidade de observar as recomendações para a prevenção de acidentes no ambiente portuário. Pode-se considerar que esse "cumprir as recomendações para a prevenção de acidentes" possui um caráter normativo, representando, portanto, uma norma a ser cumprida pelos TPAs.

\section{CONCLUSÃO}

A partir do presente estudo, pode-se concluir que os TPAs reconhecem a existência e as atribuições do PPRA, SESSTP e CPATP, bem como identificam algumas das suas funções nas citadas ações. Reconhece-se que a visão da prevenção dos agravos à Saúde do Trabalhador ainda é hegemônica, mas que pode subsidiar a Promoção da Saúde no contexto analisado, quando considerada a Saúde do Trabalhador. Como responsabilidade do campo interdisciplinar da Saúde Pública, entende-se que a enfermagem possa contribuir para a ampliação dos limites da indústria, no intuito de que ela dirija o foco ao objeto humano, no processo saúde/doença, em sua relação com o processo de trabalho, atuando na prevenção de agravos à saúde dos TPAs e realizando ações de promoção da saúde, e, ainda, desenvolvendo outros estudos na direção da enfermagem na saúde do trabalhador portuário.

Acredita-se que quando o trabalhador portuário, consciente das exposições a que está submetido no seu ambiente de trabalho, não só atua na prevenção de doenças e acidentes, mas também pode assumir a sua responsabilidade para a promoção da saúde, coparticipando na viabilização das mudanças necessárias para tornar o ambiente de trabalho menos insalubre e perigoso e, assim, promover condições saudáveis. Considerando a limitação de um estudo transversal, que não permite a avaliação adequada de fatores os quais podem ser associados ao desfecho investigado. Nesse sentido, seria interessante a realização de outros estudos, tais como os de intervenção comunitária, a fim de realizar ações e avaliar a efetividade das mesmas.

Neste sentido se destaca a importância da produção científica no ambiente portuário, ao propor divulgar o conhecimento e o fortalecimento da inserção/atuação do profissional enfermeiro nesse campo de atuação, pois, ao ser integrante das equipes multiprofissionais do SESSTP, a enfermagem possui papel fundamental, desenvolvendo a promoção da saúde por meio das ações de prevenção de doenças, visando à adoção de comportamentos mais saudáveis.

\section{AGRADECIMENTO}

Ao Conselho Nacional de Desenvolvimento Científico e Tecnológico (CNPq), pelo financiamento do projeto "Saúde do trabalhador e conhecimento sobre situações de risco: um estudo com trabalhadores portuários avulsos do município do Rio Grande-RS" (Processo n . 485227/2006-1; auxílio 2006/2008). 


\section{REFERÊNCIAS}

1. Soares JFSS, Cezar-Vaz MR, Mendoza-Sassi RA, Almeida TL, Muccillo-Baisch AL, Soares MCF et al. Percepção dos trabalhadores avulsos sobre os riscos ocupacionais no porto do Rio Grande, Rio Grande do Sul, Brasil. Cad Saúde Pública. 2008 Jun; 24(6):1251-9.

2. Santos Neto AB, Ventilari PSX. O trabalho portuário e a modernização dos portos. Curitiba (PR): Juruá; 2005.

3. Ministério do Trabalho e Emprego (BR). Portaria ${ }^{\circ}$ 26 de 29/12/1994. NR 9 Programa de prevenção de riscos ambientais. Brasília (DF): MTE. [acesso 2007 Out 10]. Disponível em: http:/ / www.ogmo-rg.com. br/leis/NR09.html

4. Ministério do Trabalho e Emprego. Portaria $n^{\circ} 53$ de 17/12/1997. NR 29 Segurança e saúde no trabalho portuário. Brasília (DF): FUNDACENTRO; 1997.

5. Candeias NMF. Conceitos de educação e de promoção em saúde: mudanças organizacionais. Rev Saúde Pública. 1997 Abr; 31(2):209-13.

6. Restrepo HE. Agenda para la ación en promoción de la salud. In: Restrepo HE, Málaga H, organizadores. Promoción dela salud: cómo construir vida saludable. Bogotá (CO): Editorial Médica Panamericana; 2001. p. 34-55

7. Cezar-Vaz MR, Cardoso LS, Bonow CA, Sant'Anna $\mathrm{CF}$, Sena J. Conhecimento clínico do enfermeiro na atenção primária à saúde: aplicação de uma matriz filosófica de análise. Texto Contexto Enferm. 2010 Jan-Mar; 19(1):17-24.
8. Ministério da Saúde (BR), Conselho Nacional de Saúde, Comissão Nacional de Ética em Pesquisa. Resolução No 196 de 10 de outubro de 1996: diretrizes e normas regulamentadoras de pesquisa envolvendo seres humanos. Brasília (DF): MS; 1996.

9. Loureiro ED. Aplicação da NR-29 no porto do Rio Grande-RS. In: Anais do II Congresso Nacional de Segurança e Saúde no Trabalho Portuário e Aquaviário [CD ROM]. Santos (SP): FUNDACENTRO; 5 a 8 de novembro de 2010.

10. Leff E. Saber ambiental: sustentabilidade, racionalidade, complexidade, poder. $2^{\mathrm{a}}$ edição. Petrópolis (RJ): Vozes, 2001.

11. Frigotto G. Trabalho, conhecimento, consciência e a educação do trabalhador: impasses teóricos e práticos. In: Minayo-Gomez C, Frigotto G, Arruda M, Arroyo M, Nosella P, organizadores. Trabalho e conhecimento: dilemas na educação do trabalhador. São Paulo: Cortez editora/autores associados; 1989. p. 13-26.

12. Garcia MLZ. A auto-atividade na educação ambiental, uma ferramenta para a ação "no fazer" humano: o ambiente transformador do trabalho portuário avulso do Rio Grande. [dissertação]. Rio Grande (RS): Universidade Federal do Rio Grande, Programa de Pós-Graduação em Educação Ambiental; 2005.

13. Valla VV. Educação, Saúde e cidadania: investigação científica e assessoria popular. Cad Saúde Pública. 1992 Mar; 8(1):30-40.
Correspondência: Marta Regina Cezar-Vaz

Universidade Federal do Rio Grande - Escola de Enfermagem Área Acadêmica da Saúde Prof. Newton Azevedo - $4^{\circ}$ andar Rua Gen. Osório, s/n ${ }^{\circ}$

96200-190 - Rio Grande, RS, Brasil

E-mail:cezarvaz@vetorial.net
Recebido: 2 de agosto de 2010

Aprovação: 5 de abril de 2011 